\title{
Preserved action of a rectus muscle after transection by an encircling solid silicone band
}

\author{
Isaac Ashkenazi, Joseph Moisseiev, Elisha Bartov, Giora Treister
}

\begin{abstract}
We present a case of anterior migration of a solid silicone band through a lateral rectus muscle. In this patient the action of the lateral rectus was preserved, and this is demonstrated photographically. The possible reasons for this rare complication and the possible mechanism by which lateral rectus activity remained intact are explained.
\end{abstract}

Exposure of implanted material after retinal detachment surgery is a known and rather common complication. It happens usually with radial buckles, but also with encircling elements. Anterior migration of an exposed solid silicone band eroding through a rectus muscle without causing disturbances in ocular motility is rare, and we believe this is the first photographic report of this rare complication of retinal detachment surgery.

Department of

Ophthalmology,

Goldschleger Eye

Institute, Sheba Medical

Center, Sackler School of

Medicine, Tel Aviv

University, Israel

I Ashkenazi

J Moisseiev

E Bartov

G Treister

Correspondence to:

Isaac Ashkenazi, $M D$,

Department of

Ophthalmology, Chaim Sheba

Medical Center, Tel-

Hashomer, Israel

Accepted for publication 29 March 1990

\section{Case report}

A 57-year-old man was referred to our department with aphakic retinal detachment in the right eye. In the year prior to his admission he underwent extracapsular cataract extraction, with vitreous loss in both eyes. This was followed by aphakic retinal detachment in both eyes, which was successfully repaired in the left eye. In the right eye he had three unsuccessful retinal detachment operations, after which he was

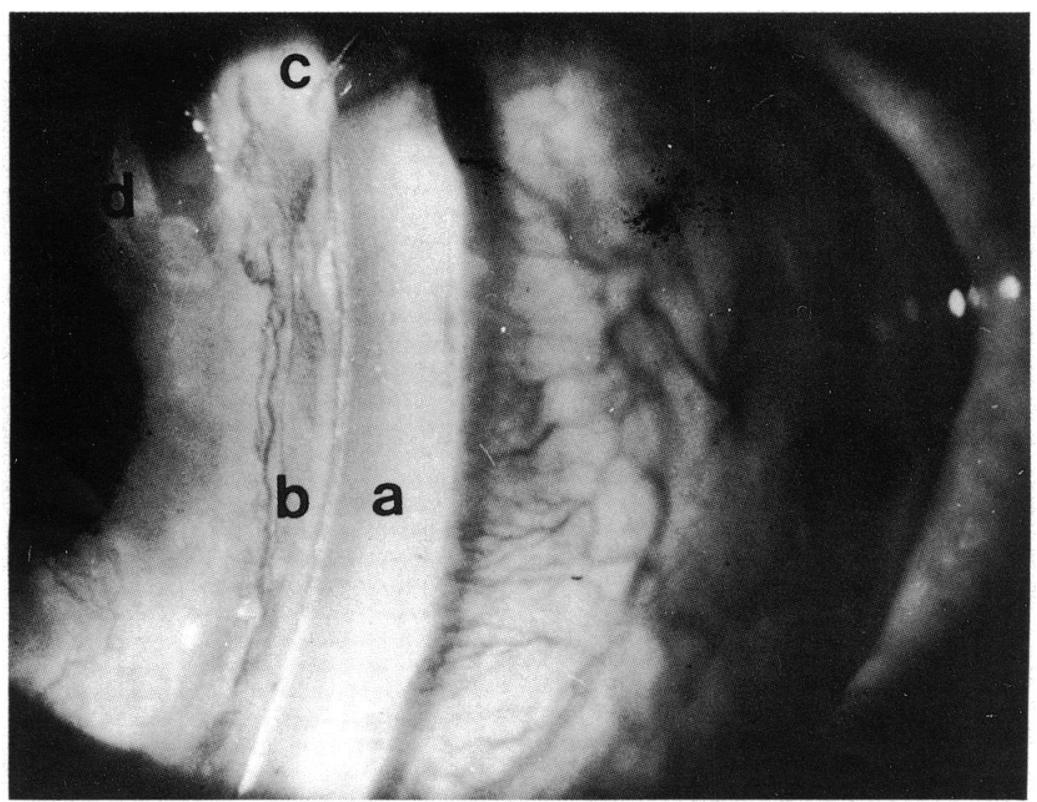

Figure 1 The $2 \mathrm{~mm}$ band (a) has migrated almost to the limbus, pushing in front of it a fold of conjunctiva $(b)$. The $5 / 0$ braided nylon scleral suture detached from the sclera and migrated with the band $(c)$. A delle was found in the adjacent cornea $(d)$.
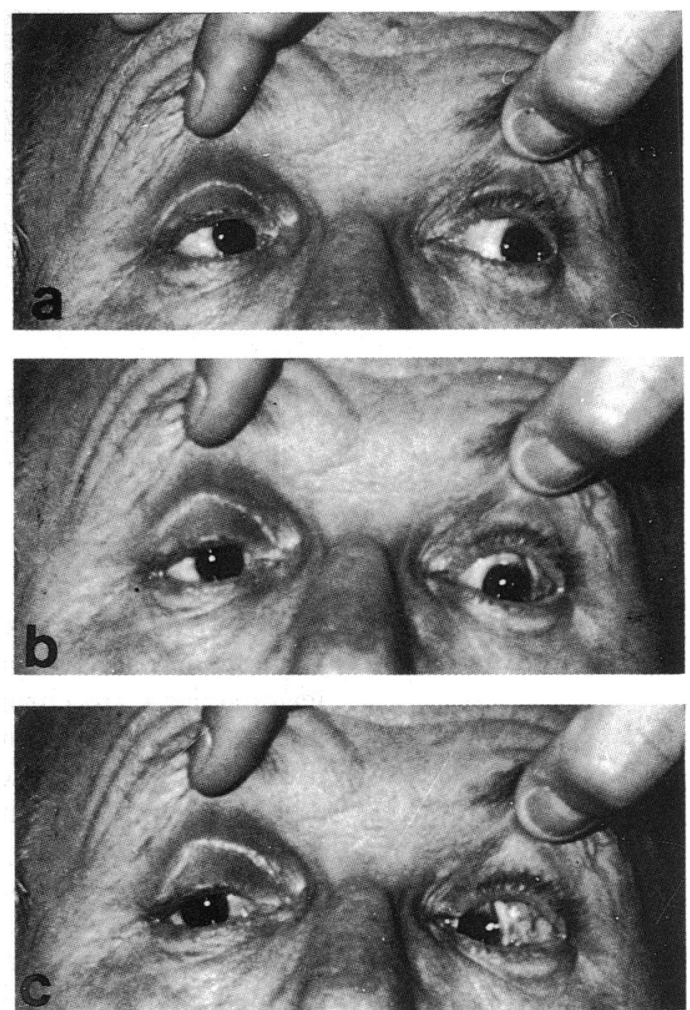

Figure 2 The eyes are shown in gaze to the left (a), primary position $(b)$, and gaze to the right $(c)$. Full range of the left lateral rectus and lack of restriction on gaze to the right are demonstrated.

referred to us. We performed vitrectomy and silicone oil injection combined with placement of a new radial sponge in the right eye, and the retina reattached.

Two months later an asymptomatic inferior retinal detachment was observed in his left eye. Vitrectomy, air exchange, removal of the previous encircling $5 \mathrm{~mm}$ silicone sponge, and placement of an encircling $2 \mathrm{~mm}$ solid silicone band and a radial silicone sponge were performed, and the retina reattached. A few weeks later the new radial sponge became exposed and was removed.

Two years after his last operation the patient returned complaining of mild discomfort in his left eye. On examination the $2 \mathrm{~mm}$ band was seen exposed on the temporal side of the limbus, pushing forward a fold of conjunctiva and causing a large delle on the corneal side of the band. Part of a torn scleral suture was seen tied to the band (Fig 1). Remarkably, lateral rectus movements were completely preserved (Fig 2). Under topical anaesthesia the exposed part of the band was resected. After a few days on topical dexamethason phosphate $0.1 \%$ four times a day and chloramphenicol $5 \%$ ointment three times a day the delle healed, and the eye became asympto- 
matic. At his most recent follow-up, in December 1988 , the retina remained attached, and he was asymptomatic.

\section{Discussion}

Anterior migration of a solid silicone band may occur if it is tight, placed anteriorly to the equator, and insufficiently anchored to the sclera. As an anterior sliding band slowly erodes through the tendon of one or more recti muscles, there is sufficient time for the muscles to reattach spontaneously with scar tissue behind the migrating element. ${ }^{1}$ We believe that such was the case with our patient. An alternative explanation might be that the band may have been placed external to the muscle. We do not believe this is possible because of the expertise of the surgeon (GT), and because the band was sutured to the sclera, as evidenced by the displaced suture attached to the band. It is inconceivable that the surgeon would not have noticed external placement of the band while he was suturing the band to the sclera close to the muscle.

It is remarkable that there was no limitation of the horizonatal eye movements, and that the lateral rectus retained its full range of movement. This indicates that the tendon was not recessed from its insertion and that the scar tissue which kept it in place did not limit its action.

1 Schepens CL. Postoperative complications: II. Problems of specific procedures. Retinal detachment and allied diseases. Philadelphia: Saunders, 1983: 1025-8. 